\title{
ON A CERTAIN IDEAL OF KÜLSHAMMER IN THE CENTRE OF A GROUP ALGEBRA
}

\author{
JOHN MURRAY
}

\begin{abstract}
Let $G$ be a finite group and let $F$ be a splitting field of characteristic $p>0$. We show that $I^{2}=E_{0}$, where $I$ is a certain ideal of the centre $Z$ of $F G$, and $E_{0}$ is the span of the block idempotents of defect zero.
\end{abstract}

Let $G$ be a finite group and let $F$ be a field of characteristic $p$. We shall assume that $F$ is a splitting field for $G$. Let $\lambda$ denote the linear map $F G \rightarrow F$, given by

$$
\lambda\left(\sum_{g \in G} a_{g} g\right)=a_{1},
$$

for $\sum_{g \in G} a_{g} g \in F G$. The map $B: F G \times F G \rightarrow F$,

$$
B(a, b)=\lambda(a b), \quad \text { for } a, b \in F G,
$$

is a nondegenerate symmetric bilinear form on $F G$. In fact $B$ is associative in the sense that

$$
B(a b, c)=B(a, b c), \quad \text { for } a, b, c \in F G .
$$

If $V$ is an $F$-subspace of $F G$, then $V^{\perp}$ will denote the dual space

$$
V^{\perp}:=\{a \in F G \mid B(a, b)=0 \text {, for all } b \in V\} .
$$

If $A$ is a subalgebra of $F G$, and $V$ is a right $A$-module, then $V^{\perp}$ is a left $A$-module. Let $K:=Z^{\perp}$, where $Z$ is the centre of $F G$. Then by the above remarks, $K$ is a $Z$-submodule of $F G$. It is clear that

$$
K=\left\{\sum_{g \in G} a_{g} g \mid \sum_{g \in \mathcal{K}} a_{g}=0, \text { for all conjugacy classes } \mathcal{K} \text { of } G\right\},
$$

as the class sums $\mathcal{K}^{+}:=\sum_{g \in \mathcal{K}} g$ form an $F$-basis for $Z$. The following lemma is due to R. Brauer [B56]:

\section{Lemma 2.}

$$
\begin{aligned}
& a \in K \\
& (a+b)^{p} \quad \equiv \quad a^{p} \in K, \\
& a^{p}+b^{p} \bmod K,
\end{aligned}
$$

for all $a, b \in F G$.

Set

and define

$$
N:= \begin{cases}4, & \text { if } p=2 \\ p, & \text { if } p \text { is an odd prime }\end{cases}
$$

$$
T:=\left\{x \in F G \mid x^{N} \in K\right\}
$$

Date: Submitted February 1, Revised March 14, 2000.

1991 Mathematics Subject Classification. 20C20. 
Then Lemma 2 implies that $T$ is a subspace of $F G$ which contains $K$. An easy argument shows that $T$ is a $Z$-submodule of $F G$. Since $T \supseteq K$, it follows that

$$
I:=T^{\perp}
$$

is an ideal of $Z$.

For each conjugacy class $\mathcal{K}$, set

$$
\Omega(\mathcal{K}):=\left\{g \in G \mid g^{N} \in \mathcal{K}\right\} .
$$

So $\Omega(\mathcal{K})$ is a union of conjugacy classes of $G$. We have the following (see (38) of [K91]):

\section{Lemma 3.}

$$
T=\left\{\sum_{g \in G} a_{g} g \mid \sum_{g \in \Omega(\mathcal{K})} a_{g}=0, \text { for all classes } \mathcal{K}\right\} .
$$

Thus $\left\{\Omega(\mathcal{K})^{+} \mid \mathcal{K}\right.$ a conjugacy class of $\left.G, \Omega(\mathcal{K}) \neq \phi\right\}$ forms a basis for $I$.

Proof. Say $a=\sum_{g \in G} a_{g} g \in F G$. Then

$$
\begin{aligned}
a \in T & \Longleftrightarrow\left(\sum_{g \in G} a_{g} g\right)^{N} \in K \text {, by definition of } T \\
& \Longleftrightarrow \sum_{g \in G} a_{g}^{N} g^{N} \in K \text {, using Lemma } 2 \\
& \Longleftrightarrow \sum_{g^{N} \in \mathcal{K}} a_{g}^{N}=0, \text { for all classes } \mathcal{K} \text {, by }(1) \\
& \Longleftrightarrow \sum_{g \in \Omega(\mathcal{K})} a_{g}=0 \text {, for all classes } \mathcal{K} \text {, as } F \text { has characteristic } p .
\end{aligned}
$$

The last statement now follows from the first.

Proposition 4. Let $z \in Z$ and suppose that $z^{N}=0$. Then $I z=z I=0$.

Proof. Let $i \in I$ and $x \in F G$. It follows from the hypothesis that $z x \in T$. Thus $B(i z, x)=B(i, z x)=0$. Since $x \in F G$ was arbitrary, the nondegeneracy of $\lambda$ implies that $i z=0$.

Let $Z_{0}$ denote the $F$-subspace of $Z$ spanned by the class sums of $p$-defect zero, and let $E_{0}$ denote the $F$-subspace of $Z_{0}$ spanned by the block idempotents of defect zero. Then $Z_{0}$ is an ideal of $Z$, using an argument due to R. Brauer. Moreover Iizuka and Watanabe [IW73] have shown that

$$
\left(Z_{0}\right)^{2}=E_{0}
$$

and

$$
Z_{0} J(F G)=0,
$$

where $J(F G)$ is the Jacobson radical of $F G$. See (1.E) of [O80] also. A proof of the following result was indicated in [M99]:

Lemma 7. $I^{2} \subseteq Z_{0}$. 
Proof. Let $\mathcal{K}, \mathcal{L}$ and $\mathcal{M}$ be classes of $G$. The coefficient of $\mathcal{M}^{+}$in $\Omega(\mathcal{K})^{+} \Omega(\mathcal{L})^{+}$is given as the cardinality, modulo $p$, of the set

$$
\Phi(\mathcal{M}):=\{(k, l) \in \Omega(\mathcal{K}) \times \Omega(\mathcal{L}) \mid k l=m\},
$$

where $m$ is a fixed element of $\mathcal{M}$. Let $D$ be a defect group of $m$. Then $D$ acts by conjugation on the pairs in $\Phi(\mathcal{M})$. So $|\Phi(\mathcal{M})| \equiv\left|\Phi_{D}(\mathcal{M})\right| \bmod p$, where

$$
\Phi_{D}(\mathcal{M}):=\{(k, l) \in(C(D) \cap \Omega(\mathcal{K})) \times(C(D) \cap \Omega(\mathcal{L})) \mid k l=m\} .
$$

Now let $\Omega(Z(D))$ be the subgroup of $Z(D)$ consisting of all $z \in Z(D)$ such that $z^{N}=1$. Then $\Omega(Z(D))$ acts freely on $\Phi_{D}(\mathcal{M})$ via

$$
(k, l) \rightarrow\left(k z, z^{-1} l\right), \quad \text { for }(k, l) \in \Phi_{D}(\mathcal{K}), \text { and } z \in \Omega(Z(D)) .
$$

It follows that $\left|\Phi_{D}(\mathcal{M})\right| \equiv 0 \bmod p$ unless $\Omega(Z(D))=\{1\} \Leftrightarrow D=\{1\}$ i.e. unless $\mathcal{M}$ has $p$-defect zero. The lemma follows.

Corollary 8. $I(I \cap J(F G))=0$ and hence $(I \cap J(F G))^{2}=0$.

Proof. Suppose that $j \in I \cap J(F G)$. Then $j \in J(F G)$, as $I \subseteq Z$. Also $j^{2} \in Z_{0}$, by Lemma 7. So $j^{3}=j\left(j^{2}\right)=0$, using (6). But then $j^{N}=j^{N-3} j^{3}=0$. Proposition 4 now implies that $I(I \cap J(F G))=0$. The equality $(I \cap J(F G))^{2}=0$ follows immediately.

We can now prove our main result.

Theorem 9. $I^{2}=E_{0}$.

Proof. Let $E$ denote the $F$-subspace of $Z$ spanned by the block idempotents. Then

$$
Z=E \oplus J
$$

as $F$-algebras, where $J=Z \cap J(F G)$ is the Jacobson radical of $Z$. Now $J$ is nil and the map $x \rightarrow x^{p}$ is an automorphism of $F$. It follows that there exists $m \geq 0$ such that $e^{p^{m}}=e$ and $j^{p^{m}}=0$, for all $e \in E$ and $j \in J$.

If $i_{1}, i_{2} \in I$, write

$$
i_{k}=e_{k}+j_{k}, \quad(k=1,2),
$$

where $e_{k} \in E$ and $j_{k} \in J$. Then $e_{k}=e_{k}^{p^{m}}+j_{k}^{p^{m}}=i_{k}^{p^{m}} \in I$. It follows that $e_{k} \in I$ and $j_{k} \in I \cap J(F G)$. So

$$
i_{1} i_{2}=e_{1} e_{2}+e_{1} j_{2}+j_{1} e_{2}+j_{1} j_{2}=e_{1} e_{2}
$$

using Corollary 8. Thus $I^{2} \subseteq E \cap Z_{0}=E_{0}$, using Lemma 7 .

The oppositite inequality $I^{2} \supseteq E_{0}$ follows from $I \supseteq Z_{0}$ and (5).

We also have:

Proposition 10. Let $\mathcal{K}$ be a p-singular class of $G$. Then $\Omega(\mathcal{K})^{+} \in J(F G)$. In particular, $\Omega(\mathcal{K})^{+} \Omega(\mathcal{L})^{+}=0$, for each class $\mathcal{L}$ of $G$.

Proof. Let $B$ be a $p$-block of $G$, with associated central character $\omega$. If $B$ has positive defect, then $\omega\left(\left(\Omega(\mathcal{K})^{+}\right)^{2}\right)=0$, using Lemma 7 , and so $\omega\left(\Omega(\mathcal{K})^{+}\right)=0$. On the other hand, if $B$ has defect zero, then $\omega\left(\Omega(\mathcal{K})^{+}\right)=0$, as $\Omega(\mathcal{K})$ is a union of $p$-singular classes. We deduce that $\Omega(\mathcal{K})^{+} \in J(F G)$. The last statement now follows from Corollary 8. 
If $g \in G$, we may write $g=g_{p} g_{p^{\prime}}=g_{p^{\prime}} g_{p}$, for a unique $p$-element $g_{p}$ and a unique $p$-regular element $g_{p^{\prime}}$. We call $g_{p}$ the $p$-part of $g$ and $g_{p^{\prime}}$ the $p$-regular part of $g$. Let $\mathcal{K}$ be a $p$-regular class of $G$. The $p$-regular section $S(\mathcal{K})$ of $G$ which contains $\mathcal{K}$ is defined as

$$
S(\mathcal{K}):=\left\{g \in G \mid g_{p^{\prime}} \in \mathcal{K}\right\}
$$

Setting $\mathcal{L}^{N}=\left\{g^{N} \mid g \in \mathcal{L}\right\}$, for each class $\mathcal{L}$ of $G$, we note that

$$
S(\mathcal{K})=\bigcup_{\mathcal{L} \subset S(\mathcal{K})} \Omega\left(\mathcal{L}^{N}\right)
$$

The $p$-regular section sums $S(\mathcal{K})^{+}$span an ideal $R$ of $Z$, known as Reynolds Ideal. We have the following chain of ideals of $Z$ :

$$
E_{0} \subseteq Z_{0} \subseteq R \subseteq I .
$$

Now $R=J(F G)^{\perp} \cap Z$, by (39) of [K91]. It follows easily that

$$
R^{2}=E_{0} .
$$

So Theorem 9 is an improvement on this fact.

Corollary 11. Suppose that $\mathcal{K}, \mathcal{L}$ are $p$-regular classes of $G$. Then

$$
S(\mathcal{K})^{+} S(\mathcal{L})^{+}=\Omega\left(\mathcal{K}^{N}\right)^{+} \Omega\left(\mathcal{L}^{N}\right)^{+}
$$

Proof. This follows from Proposition 10, and the fact that $\mathcal{K}$ and $\mathcal{L}$ are the only $p$-regular classes in $S(\mathcal{K})$ and $S(\mathcal{L})$, respectively.

The following extends results in [IW73] and [M99]:

Corollary 12. $G$ has a p-block of defect zero if and only if there exists p-regular classes $\mathcal{K}, \mathcal{L}$ of $G$ such that $\Omega(\mathcal{K})^{+} \Omega(\mathcal{L})^{+} \neq 0$, i.e. there exists $g \in G$ (necessarily of $p$-defect zero) such that the cardinality of the set

$$
\left\{(x, y) \in G \times G \mid x^{N} \in \mathcal{K}, y^{N} \in \mathcal{L}, x y=g\right\}
$$

is nonzero modulo $p$.

We now give some examples in the exceptional case where $p=2$ and $F$ has characteristic 2 . Set

Then

$$
T_{1}:=\left\{x \in F G \mid x^{2} \in K\right\} .
$$

$$
I_{1}:=T_{1}^{\perp}
$$

is an ideal of $Z$, and has as $F$-basis $\left\{\Omega_{1}(\mathcal{K})^{+}\right\}$, where $\mathcal{K}$ ranges over the conjugacy classes of $G$, and

$$
\Omega_{1}(\mathcal{K}):=\left\{g \in G \mid g^{2} \in \mathcal{K}\right\} .
$$

Although $E_{0} \subseteq I_{1}^{2} \subseteq Z_{0}$, and our results can be extended to show that $E_{0}=I_{1}^{3}$, it is not generally true that $E_{0}=\left(I_{1}\right)^{2}$. For instance, if $G=\mathcal{S}_{7}$, the symmetric group on 7-symbols, then $0=E_{0} \subset I_{1}^{2}=Z_{0}$, while if $G=M_{23}$, the Mathieu group of degree 23, then $0 \subset E_{0} \subset I_{1}^{2} \subset Z_{0}$. On the other hand, if $G=\mathcal{S}_{3}$, then $E_{0}=I_{1}^{2}=Z_{0}$.

Let $\mathcal{R}$ denote the set of elements of $G$ which have 2-defect zero and which are conjugate to their inverses. We showed in [M99] that

$$
\left(\Omega_{1}\left(1_{G}\right)^{+}\right)^{2}=\mathcal{R}^{+} .
$$

It follows that $\mathcal{R}^{+} Z \subseteq I_{1}^{2}$. We have not found an example where $\mathcal{R}^{+} Z \neq I_{1}^{2}$. 


\section{REFERENCES}

[B56] Richard Brauer, Zur Darstellungstheorie der Gruppen endlicher Ordnung Math. Zeit. 63 (1956), 406-444.

[IW73] K. Iizuka, A. Watanabe, On the number of blocks of irreducible characters of a finite group with a given defect group, Kumamoto J. Sci. (Math.) 9 (1973), 55-61.

[K91] Burkhard Külshammer, Group-theoretical descriptions of ring-theoretical invariants of group algebras, Progress in Math. 95 (1991), 425-442.

[M99] John Murray, Blocks of defect zero and products of elements of order p, J. Algebra 214 (1999), 385-399.

[O80] T. Okuyama, Some studies on group algebras, Hokkaido Mathematical J. 9 (1980), 217-221.

Mathematics Department, University College Dublin, Belfield, Dublin 4, Ireland

E-mail address: jcmurray@eircom.ie 